\title{
Fósseis da macroflora do Crato, Bacia do Araripe (Eocretáceo) depositados na coleção científica do Laboratório de Ecologia e Geociências (UFBA), em Vitória da Conquista (BA)
}

Fossils of the Crato macroflora, Araripe Basin (Early Cretaceous) deposited in the scientific collection of the Ecology and Geosciences Laboratory of (UFBA), in Vitória da Conquista (BA)

\author{
N. S. Silva; M. A. T. Dantas*; A. C. C. Rodrigues \\ Departamento de Geociências, Universidade Federal da Bahia, Instituto Multidisciplinar em Saúde BA, 45029-094, \\ Vitória da Conquista-BA, Brasil.
}

*matdantas@yahoo.com.br

(Recebido em 30 de outubro de 2020; aceito em 28 de março de 2021)

\begin{abstract}
O propósito deste trabalho foi o reconhecimento taxonômico dos fitofósseis oriundos do Membro Crato, Formação Santana da Bacia do Araripe armazenados no Laboratório de Ecologia e Geociências da UFBA, no Campus Anísio Teixeira. Os espécimes estudados totalizaram cinquenta e dois. A princípio foi feito um levantamento dos táxons de plantas fossilizadas já descritos para o Membro citado. Na sequência, o material foi fotografado e deu-se início a identificação, intentando alcançar o táxon de menor nível da classificação possível. Nesta etapa foram analisadas as morfologias dos órgãos preservados e comparadas àquelas das espécies paleobotânicas descritas em trabalhos prévios. Realizada a identificação dos espécimes estudados com suas respectivas espécies, foi feita uma descrição minuciosa de cada espécime. Apenas gimnospermas e angiospermas foram identificadas, sendo o primeiro grupo o mais representativo, especialmente pelas coníferas.
\end{abstract}

Palavras-chave: paleoflora eocretácea, Taxonomia, Coleção Depositária UFBA.

The purpose of this work was the taxonomic recognition of fossil vegetables from the Crato Member, stored in the Laboratory of Ecology and Geosciences of UFBA, at Campus Anísio Teixeira. The specimens studied add up to fifty-three two pieces. At first, there was a check on fossilized plants that have already been described to the mentioned Member. Then the material was photographed, and identification began, trying to reach the lowest level of classification. At this stage, the preserved organs morphology was analyzed and then compared to paleobotanical species detailed in several studies. After the scan was confirmed, a detailed description of the species was performed. Only gymnosperms and angiosperms were identified being the first group the most representative, particularly conifers.

Keywords: early cretaceous paleoflora, Taxonomy, UFBA Depository Collection.

\section{INTRODUÇÃO}

A Bacia do Araripe está localizada no Nordeste brasileiro, na porção central da província Borborema estendendo pelos estados do Ceará, Pernambuco e Piauí, entre as coordenadas $7^{\circ} 02^{\prime} \mathrm{S}$ $-7^{\circ} 49^{\prime} \mathrm{S}$ e $38^{\circ} 30^{\prime} \mathrm{W}-40^{\circ} 55^{\prime} \mathrm{W}$ [1]. Esta bacia ocupa uma área de $8.000 \mathrm{~km}^{2}$ [2], apresentando um padrão estratigráfico com estratos horizontais de calcário laminado, moldado por inundações flúvio-lacustres [3]. Esses ambientes sedimentares foram recebendo depósitos de organismos ao longo do tempo e favorecendo a formação de uma sucessão de camadas abundante em fósseis, com destaque para o Membro Crato, estrato de origem dos fitofósseis deste estudo.

O Membro Crato originou-se durante a Idade Aptiano (125 a 113 milhões de anos) [23] no Cretáceo Inferior e ocupa uma área de $5.500 \mathrm{~km}^{2}$. Esta unidade litoestratigráfica é constituída por calcário micrítico ou composto básico de calcita, calcário laminado de ambiente lacustre, no qual encontram-se fósseis bem preservados [4]. Dentre os fósseis coletados ressaltam-se os registros de insetos, pequenos peixes, ostracodes, pterossauros, crustáceos, quelônios e aracnídeos, além dos fitofósseis [5].

Com uma importância histórica evolutiva, as plantas fósseis têm como área do conhecimento a Paleobotânica. A compreensão das transformações climáticas, o desenvolvimento dos ecossistemas e interação dos organismos antigos podem ser conhecidos através dos estudos paleobotânicos [6, 
7]. Estas informações podem ser adquiridas devido à sensibilidade dos vegetais às mudanças climáticas que os afetam, e tornam-se testemunhas dessas transformações no ambiente terrestre [6]. Nessas análises, os órgãos vegetativos normalmente são os mais encontrados, e geralmente estão separados entre si, podendo dificultar a identificação taxonômica [8].

Neste aspecto, objetiva-se identificar taxonomicamente todos os fósseis de plantas do Laboratório de Ecologia e Geociências da Universidade Federal da Bahia, situado no Instituto Multidisciplinar de Saúde, no Campus Anísio Teixeira (UFBA/IMS/CAT), em Vitória da Conquista (BA). Pretende-se chegar até o menor nível taxonômico possível e descrever a morfologia deste material. Este estudo se justifica pela carência de informações taxonômicas dos elementos aqui analisados, tornando-se o primeiro estudo sobre as plantas fossilizadas desta Instituição. Esta pesquisa disponibilizará à comunidade acadêmica e à sociedade externa o conhecimento acerca dos fósseis de plantas, no intento de desenvolver novos estudos e preencher as lacunas sobre o entendimento da Paleobotânica neste Instituto, ressaltando sua importância.

A disseminação deste conhecimento é valiosa, podendo ser compartilhado através das coleções científicas, mantidas em museus e laboratórios universitários. Como um dos detentores de riquezas fossilíferas, o Museu de Paleontologia de Santana do Cariri, localizado no Ceará, destaca-se por armazenar principalmente os fósseis da Bacia do Araripe e representa uma unidade de divulgação científica, técnico e cultural [9]. Adicionalmente, o Laboratório de Ecologia e Geociências da Universidade Federal da Bahia, Campus Anísio Teixeira detém de uma coleção significativa de fósseis.

\section{MATERIAL E MÉTODOS}

O estudo taxonômico foi realizado no Laboratório de Ecologia e Geociências, da UFBA, na cidade de Vitória da Conquista. Este espaço, embora destinado a algumas aulas práticas, é utilizado, principalmente, para a realização de pesquisas. Neste laboratório há uma coleção científica com um acervo de quase dois mil espécimes fósseis, documentando sessenta e três ocorrências fitofossilíferas, com coletas realizadas durante trabalhos de campo. Deste total, cerca de cinquenta e dois espécimes são originários do Membro Crato, e apenas os espécimes desta localidade foram analisados.

O material fóssil dispõe de um Qrcode e numeração precedida pela sigla LEG em referência ao laboratório depositário, com exceção de poucos espécimes. Algumas peças apresentam coloração amarronzada, devido à reposição da matéria orgânica por goethita, do tipo óxido hidratado de ferro, que promove a tonalidade citada [8].

No princípio do trabalho foi realizado um levantamento bibliográfico acerca dos fósseis de plantas já identificados para o Membro Crato, destacando-se a obra "The Crato fossil Beds of Brazil" [10], somado a outros trabalhos especializados da área. Nesta etapa levou-se em consideração o estudo das partes vegetativas normalmente preservadas, como raiz, caule, folha, semente, bem como eventuais estruturas reprodutivas [11], importante para a identificação desse tipo de fóssil.

O material foi fotografado utilizando-se uma câmera DSLR Nikon D3200, para evitar o manuseio frequente dos fósseis, e assim resguardá-los de possíveis danos. Ademais, o registro fotográfico facilitou o compartilhamento com cientistas que contribuíram com sugestões para este estudo. As imagens foram a princípio comparadas aos fitofósseis ilustrados na literatura sobre a macroflora do Crato, com destaque para a Revisão da Paleoflora [12]. Adicionalmente, trabalhos de diversos estudiosos da Paleobotânica integraram o processo de identificação taxonômica preliminar. Na sequência, ao averiguar as semelhanças morfológicas foi feito um estudo sobre as características diagnósticas das espécies pressupostas.

Foram analisados o modo de disposição das folhas (filotaxia foliar) e, sempre que possível, o tipo de nervura, formato das margens, presença de brácteas e caule. Sobre a estrutura caulinar foi avaliada a existência de nó e provável gema apical. Também foram observadas estruturas reprodutivas, bem como o estado de preservação do material, com base em informações bibliográficas $[13,14,15]$. 
Os fósseis especificados foram descritos macroscopicamente e organizados nos grupos das Filicófitas, Gimnospermas e Angiospermas, conforme a classificação do Código Internacional de Nomenclatura Botânica (ICBN).

\section{RESULTADOS E DISCUSSÃO}

A coleção vegetal do Laboratório, relativa ao Membro Crato foi completamente estudada, resultando em cinquenta e dois espécimes analisados. Deste conjunto percebeu-se uma maior quantidade de gimnospermas, poucas angiospermas e nenhuma filicófita. Para o primeiro grupo, foram identificados dezenove espécimes, e quatro espécimes para o segundo. Destes espécimes, dez táxons podem ser visualizados na Figura 1.

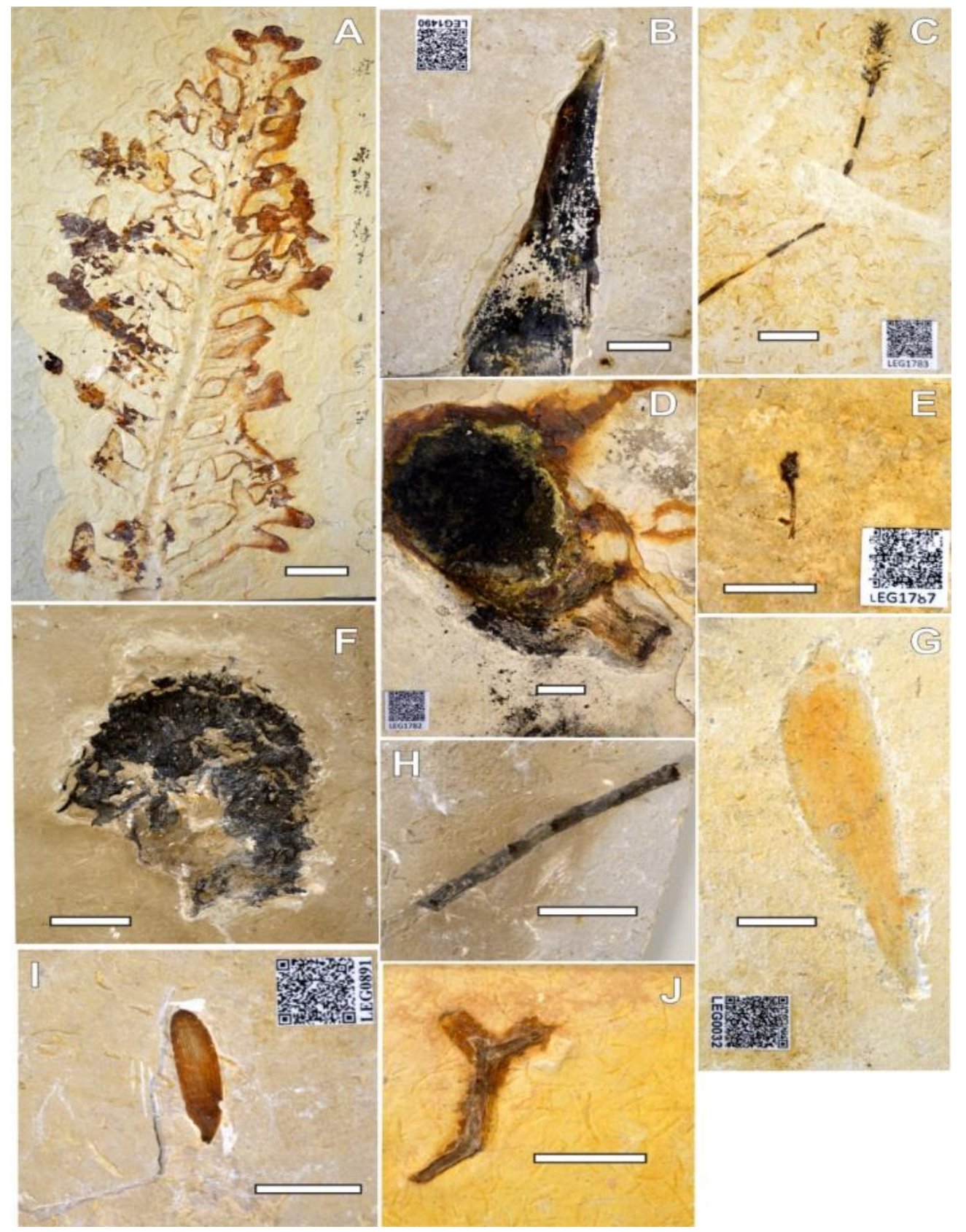

Figura 1: A. LEG 0035 Brachyphyllum obesum; B. LEG 1490 Welwitschyophyllum brasiliense; $C$. LEG 1783 Welwitschiostrobus murili; D. LEG 1782 Cone de Araucariaceae; E. LEG1787 Gnetales; F. LEG 1785 Cone de Araucariaceae; G. LEG 0032 Spixiarum kipea; H. LEG 0758 Pseudofrenelopsis; I. LEG 0891 Podozamites lanceolatus; J. LEG 0031 Duartenia araripensis. (escala $10 \mathrm{~mm}$ ). 
As dez espécies figuradas encontram-se em bom estado de fossilização, mas esta condição não pode ser atribuída a todo o conteúdo vegetal do acervo, tendo alguns aspectos a ser considerados. Observou-se neste agrupamento a presença de âmbar, troncos carbonizados, órgãos vegetativos fragmentados, e exemplares não reconhecíveis. Desses elementos foram verificados quatro âmbares, seis caules incinerados, nove espécimes inconclusivos e onze inidentificáveis (Figura 2).

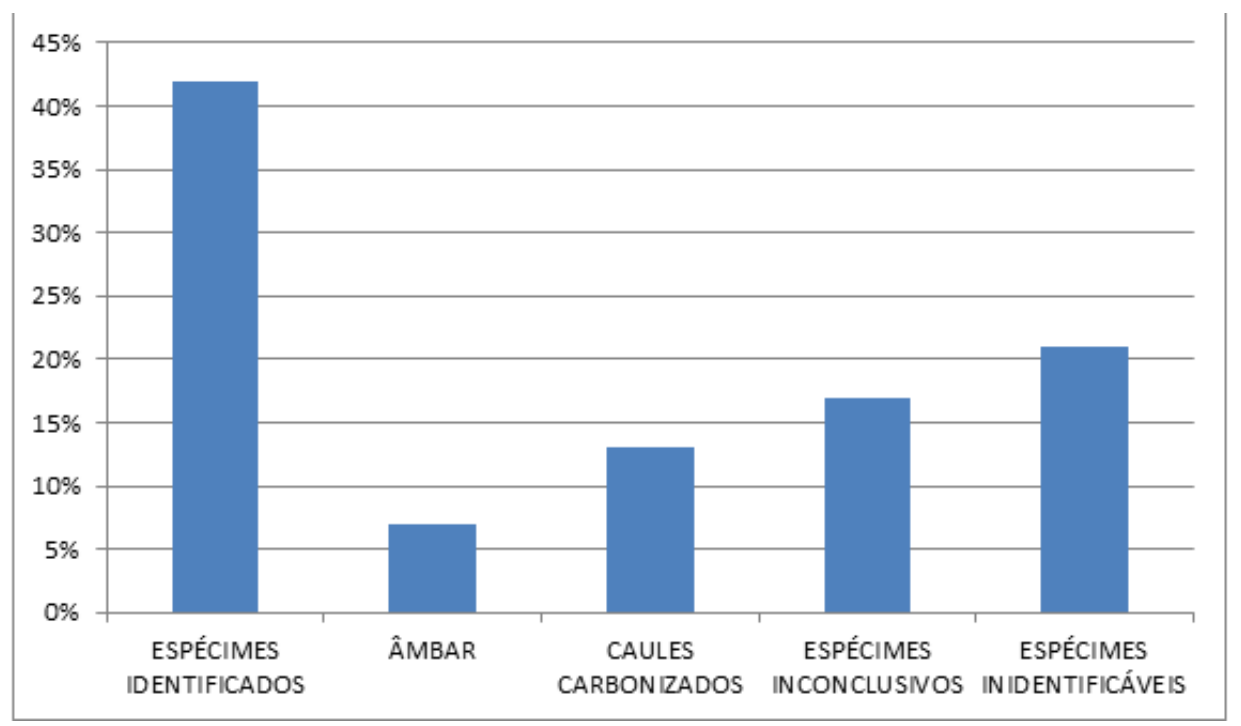

Figura 2: Caracterização completa dos fitofósseis analisados.

No esboço acima, depreende-se que $42 \%$ dos espécimes da coleção científica foram taxonomicamente reconhecidos, seguida por sete por cento de amostras correspondentes a âmbares e $13 \%$ de caules carbonizados. Perfazendo $17 \%$, os espécimes inconclusivos não foram passíveis de identificação devido à sua má preservação. Dentre esses últimos, certos exemplares apresentam alguns caracteres parecidos com fitofósseis já descritos, mas devido à sua condição citada, não foi permitida a comprovação. Na sequência, $21 \%$ dos espécimes fósseis não foram distinguidos.

Foi percebido que a maioria das espécies presentes é de gimnospermas. A ocorrência comum de fósseis gimnospérmicos na coleção deve estar relacionada ao fato de este tipo de planta vascular ter sido o mais numeroso no Eocretáceo da Bacia do Araripe [16]. Ainda nesta categoria taxonômica, as coníferas são predominantes na coleção descrita, destacando-se da Família Araucariaceae (Cones ou estróbilos e Brachyphyllum obesum), Família Cheirolepidiaceae (Pseudofrenelopsis) e cf. Cheirolepidiaceae (?) (Duartenia araripensis), e Podozamites lanceolatus. Adicionalmente, no grupo de gimnospermas encontram-se também representantes de Gnetales, como as espécies Welwitschiostrobus murili e Welwitschiophyllum brasiliense.

A descrição dos fósseis foi elaborada da seguinte forma:

\subsection{GIMNOSPERMAS ORDEM CONIFERALES}

\subsubsection{Família Araucariaceae}

\subsubsection{Estróbilo ou cone de Araucariaceae}

Na amostra catalogada como LEG 1785 (Figura 1F), há um cone preservado, pertencente à família Araucariaceae, mais especificamente ao gênero Araucariostrobus (?). Na coleção, há também outro fóssil relacionado à mesma família, o LEG 1782 (Figura 1D).

Segundo Kunzman et al. [17], a família Araucariaceae pertence a ordem Coníferales, e este tipo é retratado por um cone ovulado, lateralmente achatado, apresentando a parte distal arredondada a 
cuneada, além de possuir escamas cuneiformes no ápice. Os membros de Araucariostrobus exibem uma haste pequena, espessa e preservada em posição transversal.

A amostra catalogada por LEG 1785 encontra-se praticamente inteira, embora apresente algumas escamas-brácteas, em impressão, concentradas na base do cone. De maneira geral, este cone apresenta-se arredondado na região superior, emergindo num formato ovalado rumo à base. O estróbilo/cone é constituído por escamas-brácteas distribuídas em toda a extensão visível neste arranjo.

Em LEG 1782, nota-se um cone/estróbilo em melhor preservação, e esta característica possibilita visualizar o arranjo ondulado das escamas-brácteas, principalmente na margem próxima ao pedúnculo bem preservado. Na porção central do estróbilo, há uma concentração densa de óxido de ferro, não permitindo visualizar os detalhes das escamas-brácteas. $\mathrm{O}$ arranjo estrutural deste cone é helicoidal. O formato oval dos cones de Araucariaceae é considerado geralmente como um órgão feminino e podem ser atribuídos ao gênero cf. Araucariostrobus, porém a ausência de detalhes diagnósticos não permite o uso formal deste gênero, e por isso necessita de revisão [17].

\subsubsection{Brachyphyllum obesum Heer}

Em 1828, o gênero Brachyphyllum foi descrito por Brongniart que o caracterizou como uma conífera portadora de folhas concisas, estreitas, disposta em hélice e ramos pinados [18]. Segundo Duarte (1985) [18], a espécie Brachyphyllum obesum caracteriza-se por folhas alternas, curtas, lâmina foliar consistente/espessa e estriada. Dentre os fósseis do laboratório, três componentes foram identificados como pertencentes a esta espécie. Os elementos assim reconhecidos são: LEG 0044 com contraparte identificada por LEG 0881, LEG 0889 e LEG 0035 (Figura 1A).

Os fósseis assim identificados apresentam eixos caulinares/ramos de primeira ordem com ramos preservados, folhas com margem inteira e como descreveram Kunzmann et al. (2004) [17], as extremidades dessas estruturas caulinares neste gênero são arredondadas. As amostras da coleção apresentam algumas diferenças quanto ao padrão do limbo e sua conservação. Entre os três, LEG 0035 exibe todas as folhas, sendo o espécime mais completo. A lâmina foliar desses espécimes é simples, e varia como simples inteira, fendida e lobada [15]. No espécime LEG 0044, o limbo é simples inteiro, exceto o ápice da estrutura foliar com reentrâncias, conferindo o aspecto lobado. Essa caracterização ocorre também no espécime LEG 0889, onde mescla entre folhas sem recortes e contorno raso. Embora algumas estruturas foliares do LEG 0035 estejam desgastadas, verifica-se a presença de folhas fendidas, com delineamento mais profundo em todas as extremidades.

Todos os espécimes estão preservados em óxido de ferro, resultando em algumas diferenças na coloração. A lâmina foliácea de LEG 0035 é desbotada em toda sua extensão, exceto as bordas com coloração marrom. Este exemplar exibe nervura primária. Nas demais amostras (LEG 0044 e LEG 0889), prevalecem uma pigmentação castanha devido ao acúmulo de óxido de ferro.

\subsubsection{Família Cheirolepidiaceae}

\subsubsection{Pseudofrenelopsis Nathorst}

As amostras registradas como LEG 0757 e LEG 0758 (Figura 1H) correspondem ao gênero Pseudofrenelopsis, descrito como um ramo articulado (com nós e entrenós) [19]. Cada nó porta uma única folha, que o envolve, e forma uma sutura. As folhas de Pseudofrenelopsis são reduzidas, triangulares e arredondadas. Como destacou Watson (1977) [19], a determinação taxonômica deste grupo é marcada pela dificuldade em diferenciá-lo do gênero Frenelopsis, porque ambos apresentam lâminas foliares curtas, todavia com cutículas foliáceas desiguais. Esta diferenciação entre as duas linhagens foi ressaltada por Kunzman et al. (2004) [17], ao tipificar Frenelopsis pela presença de duas ou três folhas por nó, enquanto Pseudofrenelopsis exibe apenas uma lâmina foliar por nó. Ademais, Kunzman et al. (2004) [17] caracterizaram o segmento deste último como aberto e com sutura notável, característica não encontrada em Frenelopsis, gênero no qual os segmentos foliares envolvem-se não exibindo lacunas.

A estrutura preeminente em LEG 0757 e LEG 0758 é o caule. Nas duas amostras, o arranjo citado está completo e fixado em rocha calcária. A organização caulinar destes fósseis é do tipo 
colmo [15], sendo formada por nós e entrenós. O padrão de sutura escavada é observado em ambos os exemplares, sobretudo em LEG 0757, ao exibir esta característica em quase toda a sua extensão, enquanto em LEG 0758, os segmentos abertos situam-se próximo do ápice e da base. Nestes segmentos, não foi observado de forma nítida marcas de folha. Embora existam pontos miúdos emergindo dos nós em ambos os fósseis, não é seguro confirmar tratar-se de órgãos foliares ou gemas.

\subsubsection{Família (?)Cheirolepidiaceae}

\subsubsection{2 cf. Duartenia araripensis Mohr, Scultka, Süss \& Bernardes-de-Oliveira}

O espécime catalogado como LEG 0031 (Figura 1J) é único na coleção e está representado por um fragmento caulinar. O exemplar LEG 0031 transparece uma coloração castanha, pois toda a sua extensão foi envolvida por óxido de ferro. Na porção superior do ramo central, há uma bifurcação, porém nesta divisão, as ramificações secundárias não foram preservadas. Apesar disso, a segmentação à direita na região distal mostra um contorno circular e talvez se trate de um botão axilar, envolvido parcialmente por hematita.

Este espécime foi identificado como pertencente à espécie cf. Duartenia araripensis. De acordo com Mohr et al. 2012 [22], esta espécie pode estar ligada às Coniferales, da Família Cheirolepidiaceae, mas sua afinidade ainda é considerada incerta. Ainda segundo Mohr et al. 2012 [22], esta espécie possui um eixo caulinar lenhoso, cujo arranjo de ramos é pseudohelicoidal e anisotômico. Segundo esses autores, a espécie identificada apresenta um eixo principal sinuoso, inclinando-se para o lado esquerdo próximo da base. O eixo principal desta espécie é bifurcado e apresenta ramificações mais frágeis/delgadas, portando estruturas em forma de botões axilares. $\mathrm{O}$ lenho é denso, tipo protopináceo e composto por traqueídes com pontoações areoladas unisseriadas e raios estreitos com apenas uma pontoação com campo-cruzado podocarpoide, com folhas (quando preservadas) coriáceas semelhantes àquelas de Brachyphyllum. Entretando, no espécime analisado os caracteres como traqueídes e pontoações não foram passíveis de observação.

\subsubsection{Morfo-gênero}

\subsubsection{Podozamites lanceolatus Newberry}

Os fósseis LEG 1520 e LEG 0891 (Figura 1I) foram reconhecidos como Podozamites lanceolatus, uma conífera da família Podozamitaceae ou como Incertae sedis [17]. Ambos os espécimes consistem em somente uma folha preservada por goethita. Como retratou Lima et al. (2012) [12], nesta linhagem é comum a conservação de folhas solitárias.

Os fósseis previamente apresentados revelam um estado de preservação diferente. Em LEG 1520, observa-se apenas um fragmento mediano distal de lâmina foliar. A respeito de LEG 0891, a unidade está íntegra. Independente da conservação, estes exemplares tem um aspecto lanceolado a oblongo-lanceolado, onde o comprimento é maior em relação à largura, as margens algo paralelas, estreitando-se em direção ao topo [13]. O limbo contém nervuras consistentes e dispostas em arco de maneira sutil. No centro da folha (LEG 0891), a venação é paralela, dicotômica e dirige-se ao ápice agudo, isto é, trata-se de uma venação acródroma [12]. A parte proximal de LEG 0891 é atenuada, com estreitamento gradativo até a extremidade do órgão vegetativo, apeciolado.

\section{ORDEM GNETALES}

Em Gnetales, as folhas são opostas e partem da região basal do caule. A estrutura caulinar é cilíndrica e lenhosa, sendo o aspecto lenhoso típico de Ephedra e Gnetum. O arranjo reprodutivo em Gnetales caracteriza- se pela presença de um estróbilo helicoidal no ápice de uma haste. Segundo Mohr et al. (2007) [8], os gnetófitos são representados por Gnetum (Gnetaceae), Ephedra (Ephedraceae) e Welwitschia (Welwitschiaceae).

O estróbilo catalogado como LEG 1787 (Figura 1E) foi reconhecido como membro da ordem Gnetales. O espécime LEG 1787 apresenta um estróbilo inteiramente recoberto por óxido de ferro 
e localizado no ápice caulinar. Neste exemplar, o caule cilíndrico apresenta articulações, onde se percebe a projeção de duas folhas opostas, cujas estruturas foliares diferem na coloração, sendo uma delas recoberta praticamente por hematita, enquanto a outra apresenta-se apenas em impressão.

\subsubsection{Família Welwitschiaceae}

\subsubsection{Welwitschiostrobus murili Dilcher, Bernardes-de-Oliveira, Pons \& Lott}

Catalogados como LEG 1783 (Figura 1C) e LEG 1784, ambos os espécimes são do tipo Welwitschiostrobus murili. Estes fósseis apresentam cones reprodutivos, sustentados por eixos. Dilcher et al. (2005) [20] descreveram esta espécie como possuidora de estróbilos férteis, localizados no ápice de um eixo ou situados em gemas axilares. Mencionaram também a presença de escamas rugosas nos cones férteis.

Os fósseis encontram-se preservados em rocha calcária, estando o caule de LEG 1783 mais bem conservado, visto que em LEG 1784, o eixo apresenta-se como impressão, com apenas alguns resquícios de matéria orgânica. A integridade de ambos os cones é semelhante e apresenta uma conformação estreita e alongada. Esta estrutura tem escamas esparsas com extremidade aguda e aberta para as laterais, sugerindo maturidade.

\subsubsection{Welwitschiophyllum brasiliense Dilcher, Bernardes-de-Oliveira, Pons \& Lott}

Os espécimes LEG 1490 (Figura 1B), LEG 0884, LEG 1781 e LEG 1491 foram identificadas como pertencentes à espécie Welwitschiophyllum brasiliense. Segundo Dilcher et al. (2005) [20], esta espécie pertence à família Welwitschiaceae, dentro do grupo Gnetales. Os espécimes compartilham características como folha isobilateral, epiderme densa, formato triangular do limbo, lâmina foliar alongada, base curva ou semicircular e nervuras paralelas.

Os fósseis referentes à espécie encontram-se preservados em rocha calcária, e cada espécime exibe uma folha isolada. A estrutura foliar em todos os espécimes está praticamente inteira e exibe uma coloração marrom, diversificando apenas no LEG 0884 por apresentar uma tonalidade mais clara. O limbo dos quatro espécimes é coriáceo e apresenta veias paralelas fina. A configuração triangular das folhas mencionada por Dilcher et al. (2005) [20], reconhecida pelo ápice agudo e base mais extensa e envolvente, foi observada nos espécimes LEG 1490 e LEG 1781.

\subsection{ANGIOSPERMA MONOCOTILEDÔNEA}

\subsubsection{Spixiarum kipea Coiffard, Mohr \& Bernardes-de-Oliveira}

Exemplar único da coleção paleobotânica do Laboratório está representado por uma folha fóssil. Este espécime, referenciado como LEG 0032 (Figura 1G), apresenta uma morfologia intacta. Segundo Coiffard et al. (2013) [21], esta espécie é considerada uma monocotiledônea, provavelmente uma proto-Araceae. Em contrapartida, em Spixiarum kipea além da veia média, observam-se também veias transversais cruzando as veias equidistantes.

No espécime LEG 0032, a folha contém margem inteira e aspecto lanceolado. Este formato caracteriza-se por alongar-se mais verticalmente e exibe uma constrição rumo ao ápice [13]. O ápice apresenta-se praticamente como impressão, e dispõe de um formato arredondado e acuminado. A base também é cuneada. A lâmina foliar mostra uma descoloração e distingue-se a presença de nervuras finas paralelas. A venação tem arranjo curvinérveo-acródromo. As nervuras estão delineadas em forma paralela sem dicotomias.

\section{CONCLUSÃO}

Os agrupamentos biológicos, como verdadeiro patrimônio natural, permitem o reconhecimento da biodiversidade, e através dos conjuntos científicos preservados em instituições depositárias, temse a conservação das fontes históricas, ambientais, ecológicas, podendo estes saberes serem 
compartilhados com toda a comunidade científica e popular. $\mathrm{O}$ acesso a este conhecimento proporciona o avanço científico, ao promover pesquisas inovadoras e aplicáveis às áreas de desenvolvimento ambiental e social.

Compreende-se que através dos estudos dos fósseis é possível entender as transformações que acometeram o planeta Terra, bem como discernir o tipo de clima, relevo e ecologia num intervalo específico do tempo geológico. As coleções fósseis mantidas nos museus e laboratórios assumem um papel importante no armazenamento e preservação desse material. Essas instituições de pesquisas são as maiores detentoras deste conhecimento, e as principais as principais responsáveis por sua divulgação.

A identificação taxonômica é primordial, sobretudo para uma coleção científica. Os resultados deste estudo taxonômico revelam que na coleção fitofossilífera do Crato depositada no LEG, as gimnospermas são o grupo com maior representatividade, sendo a excelente preservação de seus órgãos (vegetativos e estruturas reprodutivas) que permite seu estudo mais detalhado. Já a frequência de angiospermas nesta assembleia é baixa.

As amostras não mencionadas da coleção necessitam de estudos mais profundos sobre a morfologia e anatomia em geral. Uma vez que, a maioria dos fitofósseis encontra-se identificada preliminarmente, esta condição facilitará o reconhecimento dos espécimes de fitofósseis da coleção, antes não identificados, possibilitando aos acadêmicos da Instituição e da comunidade externa acesso às informações de sistemática dessas amostras, permitindo aprofundar as pesquisas na área.

\section{AGRADECIMENTOS}

Os autores agradecem ao Laboratório de Ecologia e Geociências da Universidade Federal da Bahia, Campus Anísio Teixeira pela permissão e incentivo à esta pesquisa. Agradecem também às pesquisadoras Paula Sucerquia e Flaviana de Lima pelo apoio na identificação dos fósseis.

\section{REFERÊNCIAS BIBLIOGRÁFICAS}

1. Bernardes-de-Oliveira MEC, Dilcher D, França Barreto AM, Ricardi-Branco F, Mohr BAR, CastroFernandes MC. La flora del Miembro Crato, Formación Santana Cretácico Temprano de la Cuenca de Araripe, Noreste del Brasil. In: X Congresso Geológico Chileno; 2003; Concepción, Chile. [place unknown: publisher 2003.2 Disponível em: https://biblioserver.sernageomin.cl/opac/DataFiles/BernardesdeOliveiraM_et_al.pdf

2. Coimbra JC, Arai M, Carreño AL. Biostratigraphy of Lower Cretaceous microfossils from the Araripe basin, northeasterm Brazil. Geobio. 2002 Nov-Dez;35(6):687-698, doi: 10.1016/s0016-6995(02)000827

3. Soares PC, Landim PMB, Fúlfaro VJ. Tectonic cycles and sedimentary sequences in the Brazilian intracratonic basins. Geological Society of American Bulletin. 1978 Fev;89(2):181-191, doi: 10.1130/0016-7606(1978)89<181:TCASSI>2.0.CO;2

4. Lima JF. Taxonomia e posicionamento estratigráfico de vegetais fósseis da Formação Romualdo, Cretáceo Inferior da Bacia do Araripe [dissertação de mestrado]. Recife (PE): Programa de Pós-graduação em Geociências, Universidade Federal de Pernambuco; 2013. 75 p.

5. Assine ML. Bacia do Araripe. Boletim de Geociências - Petrobrás. 2007 Mai;15(2):371-389.

6. Guerra- Soomer M. Paleobotânica: Porque não só de dinossauro vive a Paleontologia. ClickCiência. 2011 Fev;23. Disponível em: http://www.clickciencia.ufscar.br/portal/edicao23/materia3_detalhe.php

7. Barros OA, Pontes AP, Batista MEP, Silva JH, Saraiva AA. Aspectos paleoecológicos da macrofauna dos folhelhos associados à camada de gipsita, Bacia do Araripe. 2016 Dez;26(2):147-156, doi: 10.18190/1980-8208/estudosgeologicos.v26n2p147-156

8. The macrophyte flora of the Crato Formation. In: Martill DM, Bechly G, Loveridge RF, editors. The Crato fossil Beds of Brazil. Window into an Ancient world. 1th ed. Vol. 1. New York (US): Cambridge University Press; e2007.p.537-565.

9. Ferreira JS, Bantim RAM, Lima FJ, Sayão JM, Saraiva, AAF. Desvendando a coleção de Pterossauros do Museu de Paleontologia da Universidade Regional do Cariri. Caderno de Cultura e Ciência. 2016 Out;15(1):57-71, doi: 10.14295/cad.cult.cienc.v15i1.942 
10. Martill DM, Loveridge RF. The Crato fossil Beds of Brazil. New York (US): Cambridge University Press; 2007. $674 \mathrm{p}$.

11. Ricardi- Branco F. Jazigos fitofossilíferos do Brasil. In: Carvalho IS, editor. Paleontologia. 3th ed. Vol. 1. Rio de Janeiro (RJ): Interciência; e2010. p. 585-593.

12. Lima JF, Saraiva AAF, Sayão JM. Revisão da Paleoflora das formações Missão Velha, Crato e Romualdo, Bacia do Araripe, Nordeste. Estudos Geológicos. 2012 Jun;22(1):99-115, doi: 10.18190/19808208/estudosgeologicos.v22n1p99-115.

13. Gonçalves EG, Lorenzi H. Morfologia vegetal. Organografia e dicionário ilustrado de morfologia das plantas vasculares. São Paulo: Instituto Plantarum de Estudos da Flora Ltda; 2007. 225 p.

14. Silva RCVM, Silva ASL, Fernandes MM, Margalho LF. Brasília (DF): Embrapa Amazônia Oriental; 2014. 26p.

15. Almeida M, Almeida CV. Morfologia da folha de plantas com sementes. Coleção Botânica 3. Piracicaba (SP): ESALQ/USP; 2018. 111 p. doi: 10.11606/9788586481642.

16. Mohr BAR, Friis EM. Early Angiosperms from the Lower Cretaceous Crato Formation (Brazil), a preliminary report. International J Plant Sci. 2000 Nov;161(6):155-166, doi: 10.1086/317580.

17. Kunzman L, Mohr BAR, Bernardes-de-Oliveira ME. Gymnosperms from the Lower Cretaceous Crato Formation (Brazil). I. Araucariaceae and Lindleycladus (Incertae sedis). Mitteilung aus dem Museum fur Naturkunde Berlin, Geowissenschaftliche Reihe. 2004 Abr;7(1):155-174, doi: 10.1002/mmng.20040070109

18. Duarte L. Vegetais fósseis da Chapada do Araripe, Brasil. Coletânea de Trabalhos Paleontológicos, DNPM. 1985; 27 (2): 585-617.

19. Watson J. Some Lower Cretaceous conifers of the Cheirolepidiaceae from the U.S.A and England. Palaeontology. 1977;20(4):715-749.

20. Dilcher D, Bernardes-de-Oliveira ME, Pons D, Lott TA. Welwitschiaceae from the Lower Cretaceous of Northeastern Brazil. American Journal of Botany. 2005 Ago;92(8):1294-1310, doi: 10.3732/ajb.92.8.1294

21. Coiffard C, Mohr BAR, Bernardes-de-Oliveira ME. The Early Cretaceous Aroid, Spixiarum kipea gen. et sp. nov., and implications on early dispersal and ecology of basal monocots. Taxon, Paleobotany. 2013 Out;62(5):997-1008, doi: 10.12705/625.21

22. Mohr BAR, Shultka S, Süss H, Bernardes-de-Oliveira ME. A new drought resistant gymnosperm táxon Duartenia araripensis gen. nov. et sp. Nov. (Cheirolepidiaceae?) from the Early Cretaceous of Northern Gondwana. Palaeontographica, Abt B: Paleobotany- Palaeophytology. 2012;289(1-3):1-25, doi: $10.1127 / \mathrm{palb} / 289 / 2012 / 1$

23. Cohen, KM, Finney SC, Gibbard PL, Fan J-X. (2013; updated) The ICS International Chronostratigraphic Chart. $\quad$ Episodes 36:199-204. Disponível em: http://www.stratigraphy.org/ICSchart/ChronostratChart2020-03.pdf 